Relations industrielles

Industrial Relations

\title{
Une cinquième année
}

\section{La Rédaction}

Volume 5, numéro 1, septembre-octobre 1949

URI : https://id.erudit.org/iderudit/1023264ar

DOI : https://doi.org/10.7202/1023264ar

Aller au sommaire du numéro

Éditeur(s)

Département des relations industrielles de l’Université Laval

ISSN

0034-379X (imprimé)

1703-8138 (numérique)

Découvrir la revue

Citer cet article

La Rédaction (1949). Une cinquième année. Relations industrielles / Industrial Relations, 5(1), 1-1. https://doi.org/10.7202/1023264ar

Tous droits réservés (C Département des relations industrielles de l’Université Laval, 1949
Ce document est protégé par la loi sur le droit d'auteur. L’utilisation des services d'Érudit (y compris la reproduction) est assujettie à sa politique d'utilisation que vous pouvez consulter en ligne.

https://apropos.erudit.org/fr/usagers/politique-dutilisation/ 


\section{Bulletin des relations industrielles}

Volume 5, numéro 1

QUÉBEC

septembre-octobre 1949

\section{UNE CINQUIÈME ANNÉE}

Comme beaucoup d'oeuvres appelées à remplir une fonction éducative et sociale désintéressée, le Bulletin des relations industrielles de l'Université Laval a commencé péniblement et a progressé laborieusement. Malgré tout, le seul fait qu'il ait réussi à tenir le coup pendant quatre années est un témoignage fort éloquent de sa vitalité et un signe certain de son utilité grandissante. Au cours de cette période en effet, on veut bien nous assurer qu'il a rendu des services inappréciables.

Fort des encouragements reçus dans le passé, le Bulletin entreprend sa cinquième année dans une toilette nouvelle et avec une volonté accrue de remplir de mieux en mieux sa mission. Jusqu'à aujourd'hui il s'est présenté à ses lecteurs sous une apparence plutôt sobre. Dans le but de plaire davantage et de donner à cette publication une allure plus conforme au dynamisme de ses idées comme aux exigences de la publicité moderne, le Département des relations industrielles a décidé de rajeunir et d'égayer sa présentation en adoptant une couverture appropriée.

Afin d'accorder plus de soin au choix des articles et d'agrandir le cercle des collaborateurs, tout en apportant une attention spéciale à la préparation technique du Bulletin, un comité de rédaction a été formé comme suit: M. l'abbé Gérard Dion, directeur, M. Gaston Cholette, secrétaire de la rédaction, MM. Jean Gagné et Denys Dion, conseillers. Ce comité a déjà apporté des améliorations qui contribueront singulièrement à rehausser la valeur et l'influence du Bulletin. Une chronique des revues est inaugurée dans le présent numéro. Au cours des prochains mois, d'autres rubriques seront ajoutées: une chronique des livres, une section de recherches, une autre consacrée à l'information, un espace réservé zux remarques des lecteurs, etc.

La plupart des changements adoptés auront. pour résultat de faire du Bulletin un moyen d'information plus efficace. Cette orientation n'indique nullement que les articles doctrinaux seront moins nombreux ou que l'espace qui leur sera réservé sera moins considérable. Le Bulletin trahirait sa mission s'il agissait ainsi, car il a pour but principal d'interpréter la doctrine sociale de l'Eglise et de commenter, à la lumière des textes pontificaux, les principaux événements qui surgissent dans le monde des relations du travail. Son caractère doctrinal ne sera donc aucunement diminué et sera même accentué.

Il y a deux ans, la plupart des articles normatifs ont porté sur les réformes de structure dans l'entreprise. Etant donné le grand intérêt soulevé par ces travaux et les réactions qu'ils ont provoquées, d'autres études seront préparées au cours de l'année qui commence sur le même sujet. Si le Bulletin attache tant d'importance da ce thème, c'est qu'il lui apparait comme l'un des principaux sur lesquels il importe d'attirer l'attention et de provoguer de sérieuses méditations, non seulement da cause de sa complexité, mais surtout d̀ cause de l'urgence de son application pour la rénovation chrétienne de la société.

La Rédaction espère que les lecteurs seront satisfaits de ces initiatives et lui en sauront gré es parcourant attentivement le Bulletin et en lui assurant une plus large diffusion.

La Rúdaction

Les lecteurs sont priés de prendre note que le premier numéro de chaque Volume du Bulletin ne paraîtra plus. en septembre mais en octobre. C'est ainsi que le présent numéro est le premier du Volume 5. 